\title{
Epidemiological Investigation of Salmonella enterica Isolates in Children with Diarrhea in Chengdu, China
}

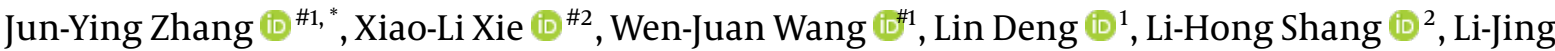 \\ Xiong (iD) ${ }^{2}$ and Pei-Pei Song (iD ${ }^{1}$ \\ ${ }^{1}$ Department of Clinical Laboratory, Chengdu Women's and Children's Central Hospital, School of Medicine, University of Electronic Science and Technology of China, \\ Chengdu 611731, China \\ ${ }^{2}$ Department of Pediatric Gastroenterology, Chengdu Women's and Children's Central Hospital, School of Medicine, University of Electronic Science and Technology of \\ China, Chengdu 611731, China \\ "Corresponding author: Department of Clinical Laboratory, Chengdu Women's and Children's Central Hospital, School of Medicine, University of Electronic Science and \\ Technology of China, Chengdu 611731, China. Email: 2867074583@qq.com
}

\# These authors are contributed equally as the first author.

Received 2021 August 27; Revised 2021 October 06; Accepted 2021 October 06.

\begin{abstract}
Background: Children with the immature intestinal immune system are prone to Salmonella infection through the fecal-oral route causing diarrhea. Non-typhoid Salmonella (NTS) is difficult to treat and eliminate due to its zoonosis. Salmonella typhi, including typhoid and paratyphoid $A, B$, and $C$, only infect humans and cause invasive infectious diseases. Salmonella typhi infection is serious and requires antibiotic treatment. The bacterial resistance caused by conventional antibacterial drugs brings great difficulties to treatment.

Objectives: This study aimed to investigate the epidemiology of S. enterica in children with diarrhea in Chengdu, China.

Methods: Fresh stool specimens or rectal swabs from 6656 children aged 1 day to 13 years with diarrhea were collected, cultured, identified, and tested for antimicrobial susceptibility. Analytical Profile index $20 \mathrm{E}$ was used for biochemical identification, and the Kirby-Bauer method was used for the bacterial sensitivity test. The whole process was conducted in accordance with the fourth edition of the National Clinical Examination procedures, and the drug sensitivity test was conducted in accordance with the Clinical and Laboratory Standards Institute 2020 guidelines.

Results: A total of 649 Salmonella strains were isolated from 6656 children with suspected Salmonella infection, among which the isolation rates of NTS and S. typhi were $8.92 \%$ and $0.83 \%$, respectively. The infection rate of S. typhimurium was the highest every year (74.88\%). Salmonella infections are on the rise, especially typhimurium, Dublin, Typhi, and London. Paratyphi is unstable, presenting a phenomenon of transition and replacement (the male to female ratio:1.12:1). The infection rate was the lowest within 1 day and 6 months $(\mathrm{P}<0.05)$. Salmonella mainly infected children under 3 years of age, and the positive rate was reported as $88.29 \%$. Within June-September, the infection rate of Salmonella was the highest, with a positive rate of $72.73 \%$. The isolated 649 Salmonella strains had good susceptibility to cefotaxime and ciprofloxacin ( $87.67 \%$ and $79.20 \%$, respectively), almost no susceptibility to ampicillin, and a drug resistance rate of $92.91 \%$.

Conclusions: The typhoid and paratyphoid vaccines should be considered together, and vaccines should focus on children under 3 years of age. Antibiotics should be rationally selected according to the drug sensitivity test and disease condition.
\end{abstract}

Keywords: Child, Salmonella enterica, Salmonella Infections, Diarrhea, Serogroup, Microbial Sensitivity Tests

\section{Background}

Salmonella enterica consists of more than 2600 different serovars that can be divided into typhoidal and nontyphoid Salmonella(NTS) serovars. Although typhoid and NTS are the same species, they elicit very different diseases in humans due to different mechanisms (1). Salmonellosis is a global challenge to public health. The NTS causes globally at least 93.8 million cases of gastroenteritis each year, with 155,000 deaths (2). The NTS infection is a consider- able burden in both developed and developing countries. Salmonella typhi causes an estimated 11.9 to 26.9 million enteric fever cases, with 129,000 to 216,510 deaths worldwide each year $(3,4)$. The enteric fever burden is mainly concentrated in low- and middle-income countries, mainly in Asia and Africa (5-7).

Salmonella tends to affect mostly sickly neonates, especially newborns under 3 years of age, due to their immature gut immune systems (8). Salmonella has a high inci- 
dence in African children, partly due to risk factors, such as human immunodeficiency virus, severe malaria, anemia, and malnutrition (9). When fungicides are used to prevent and treat disease, bacterial cells have various mechanisms to evade the toxic activity of fungicides, thereby reducing their effectiveness and ultimately leading to increased drug resistance (10). Antimicrobial resistance in Salmonella is another serious public health problem that seriously affects the successful treatment of Salmonella infections.

The surveillance of serotyping is a frequent component of a public health response to the challenget of salmonellosis (11). China is a rapidly developing country with a population of over 1.3 billion. For public health purposes, China has established its own Salmonella surveillance site and China Antimicrobial Surveillance Network (CHINET). Moreover, CHINET show that salmonella infection is increasing in China (12) and a national survey show that the infection rate of typhoid is decreasing (13).

Chengdu Women's and Children's Central Hospital is a regional third-grade hospital, the largest specialized hospital for women and children in Chengdu, China, and a sentinel of diarrhea syndrome surveillance in the National Center for Disease Control and Prevention. The cases cover the downtown and surrounding areas of Chengdu, which is of great significance to the study of the prevalence of Salmonella infection in children with diarrhea in Chengdu. This study was a detailed investigation performed on Salmonella.

\section{Objectives}

This study by the investigation of the epidemiological characteristics of Salmonella infection of children with diarrhea, especially in terms of gender, age, season distribution, serotypes, and drug susceptibility, aimed to provide a theoretical basis for the prevention and control of the occurrence of this disease.

\section{Methods}

\subsection{Study Subjects}

A child with three or more defecations per day accompanied by changes in feces character such as loose stools, mucous stools, or mucous pus blood stools were diagnosed diarrhea. This diarrheal child with either of the two characteristics, namely (1) fever $>38^{\circ} \mathrm{C}$, accompanied by chills, headache, and fatigue, and (2) abdominal pain, nausea, vomiting, and other symptoms, was considered the suspected case of Salmonella infection. The exclusion criteria were children with diarrhea presented with watery stools, without red blood cells, white blood cells (or) pus cells in routine stool test, with low blood image, and with mild symptoms. A total of 2543 cases (male: 1325 and female: 1218) were excluded from the study.

This study was a retrospective epidemiological study of 6,656 hospitalized children (male: 3767 and female: 2889) with diarrhea suspected of Salmonella infection in the Department of Pediatric Gastroenterology, Women's and Children's Central Hospital of Chengdu, China, within January 2018 to December 2020. The patients' age was within the range of 1 day to 13 years with a mean of $2.5 \pm$ 1 years and was divided into five age groups, including 0 - 6 months, 7-12 months, 1 - 3 years, 4 - 6 years, and $7-13$ years.

\subsection{Instruments and Reagents}

Incubation box, agar plates (Zhengzhou Antu Bioengineering Co., Ltd, China): blood agar plate, MacConkey agar, Eosin Methylene Blue agar, Salmonella-Shigella agar, and Mueller-Hinton Gram-negative enrichment broth, Kliger's Iron agar, and motive indole urea (Hangzhou Binhe Microbial Reagent Co., Ltd, China), Analytical Profile index (API) 20E (bioMerieux, France), Salmonella Diagnostic Sera (Ningbo Tianrun Biological Pharmaceutical Co., Ltd, China), and pills, namely ampicillin, sulfamethoxazole, ciprofloxacin, and cefotaxime, (Hangzhou Binhe Microbial Reagent Co., Ltd, China).

\subsection{Bacterial Culture}

A trained and experienced nurse collected the fresh stool specimens or rectal swabs from a child with diarrhea who was a suspected case of Salmonella infection and had not taken antibiotics in the past 5 days, placed them in a sterile container, and sent them immediately to the Microbiology Laboratory of Women's and Children's Central Hospital. The specimens were inoculated within 2 hours onto three media, including blood agar plate, Mac-Conkey agar or Eosin Methylene Blue agar, and Salmonella-Shigella agar, using a calibrated inoculating loop in the streak plate method. Then, the media were then incubated aerobically at $35^{\circ} \mathrm{C}$ for 18 - 24 hours. The samples with lower bacteria were inoculated with Mueller-Hinton Gram-negative enrichment broth for 24 hours.

\subsection{Bacterial Identification}

According to the regulations (14), a single black suspicious colony from the Salmonella-Shigella agar plate was selected and inoculated on Kliger's Iron agar and motive indole urea cultures and incubated for 24 hours. If the bacterium was red on the skew of Kliger's Iron agar, yellow on its bottom, with black gas (i.e., positive for hydrogen sulfide), and dynamic positive on motive indole urea, 
then the bacteria were suspected Salmonella. Suspected Salmonella was identified by API 20E. Once biochemical results confirmed Salmonella, a Salmonella diagnostic sera agglutination test was performed on the confirmed bacteria using a slide method. In the serotype experiment, the slide agglutination experiment of the A-F polyvalent serum of Salmonella was carried out. If the agglutination occurred, $\mathrm{O}$ and $\mathrm{H}$ factor serum was used to group and type, respectively. When A-F polyvalent was not agglutinated, the Vi agglutination experiment was carried out. If $\mathrm{Vi}$ antigen was positive, Vi was destroyed by boiling water bath $\left(100^{\circ} \mathrm{C}\right.$ for 15 - 30 minutes), and the serum agglutination test was performed again after cooling. If A-F polyvalent was not agglutinated and Vi antigen was negative, it might be that this strain was not Salmonella or other Salmonella strains other than A-F and needed to be sent to Laboratory of Microbiology, Sichuan Provincial People's Hospital in China for identification. The final test results were compared to the antigen table of Salmonella Kauffmann-White classification, and the serotype was retrieved.

\subsection{Antimicrobial Susceptibility Test}

The Kirby-Bauer method recommended by the World Health Organization was used in drug sensitivity tests. Four antibacterial drugs were selected according to the Clinical and Laboratory Standards Institute (CLSI) and regulations (14). These four pills were ampicillin, sulfamethoxazole, ciprofloxacin, and cefotaxime. MuellerHinton with a thickness of $4 \mathrm{~mm}$ was used, and the concentration of the microbial liquid was corrected to 0.5 Mcfarland standard. The spread plate method was used to inoculum the liquid. After the incubation of the Mueller-Hinton plate at an air temperature of $35^{\circ} \mathrm{C}$ for 16 - 18 hours, the diameter of the antibacterial ring was measured according to CLSI standards, and the judgment results of susceptible $(\mathrm{S})$, intermediate (I), and resistant (R) were obtained. The interpretation of breakpoints was based on the CLSI standard (http://www.clsi.org/clinical breakpoints/).

For controlling the quality of the experiment, the standard strain ATCC25922 was used for quality control to monitor the results of the experimental strain. The experimental results were analyzed by WHONET statistical software (version 5.6).

\subsection{Statistical Analysis}

SPSS statistics version 22 (SPSS Inc. Chicago, IL, USA) was used to build the database and analyze the data. Furthermore, the chi-square test was used to compare the sample rate between the groups. Two-sided P-values of less than 0.05 were considered statistically significant.

\section{Results}

\subsection{Serotype Distribution}

In total, 649 Salmonella strains were successfully isolated from the stool specimens of 6656 children with diarrhea in Chengdu in the past 3 years, with a detection rate was of 9.75\%. Moreover, 19 serotypes were isolated, among which the detection rates of NTS and S. typhi were $8.92 \%$ (594/6656) and 0.83\% (55/6656), respectively. The five most frequent serotypes were typhimurium, paratyphi, Dublin, typhi, and London, with a positive rate of 90.29\% (586/649). Within 2018 - 2020, the infection rate of S. typhimurium was the highest $(n=486 ; 74.88 \%$ ) every year; nevertheless, the infection rate of S. enteritis was low $(\mathrm{n}=7.0 ; 1.08 \%)$. Salmonella typhi and paratyphi have been detected annually at low levels. The positive rates of typhi, paratyphi $A$, paratyphi $B$, and paratyphi $C$ were $4.16 \%, 0.31 \%, 3.08 \%$, and $0.92 \%$, respectively. paratyphi is unstable, presenting a phenomenon of transition and replacement. Within 2018-2020, Salmonella infections were on the rise, especially typhimurium, Dublin, typhi, and London. In 2020, all the serotypes reached the highest level, new outbreaks of Sick Cattle appeared, and Stanley suddenly increased (Table 1).

\subsection{Gender Distribution}

The positive rates of male and female subjects were $52.85 \%$ (343/649) and 47.15\% (306/649), respectively, (the male to female ratio: 1.12:1)) with no statistical significance (Table 2).

\subsection{Age Distribution}

Among 6656 children with diarrhea, the isolation rate within the age group of three years was 9.60\% (573/5970), and the positive rate was $88.29 \%$ (573/649). In the five age groups, the isolation rate of the 0-6-month group was lower than that in any other four age groups, with statistical significance $(\mathrm{P}<0.05)$. The number of Salmonella infections in children with diarrhea is declining as they become older. The isolation rate at the 7-13-year group was lower than those of other age groups, including 7-12 months, 1-3 years, and 4 - 7 years, with statistical significance $(\mathrm{P}<0.05$; Table 3).

\subsection{Monthly Distribution}

Within January-June, the number of Salmonella isolates increased, and the peak period of diseases fell within JuneSeptember. The positive rate in the four months was $72.73 \%$ (472/649) (Table 4 and Figure 1). 


\begin{tabular}{|c|c|c|c|c|c|}
\hline Salmonella Serovars & 2018 & 2019 & 2020 & In Total & $\%$ of Total Isolates \\
\hline typhimurium & 39 & 152 & 295 & 486 & 74.88 \\
\hline Dublin & 0 & 10 & 18 & 28 & 4.31 \\
\hline London & 0 & 2 & 15 & 17 & 2.62 \\
\hline Sick Cattle & 0 & 0 & 15 & 15 & 2.31 \\
\hline Stanley & 1 & 0 & 11 & 12 & 1.85 \\
\hline Enteritidis & 1 & 3 & 3 & 7 & 1.08 \\
\hline Thompson & 2 & 1 & 2 & 5 & 0.77 \\
\hline Manhattan & 0 & 1 & 4 & 5 & 0.77 \\
\hline Newland & 0 & 1 & 3 & 4 & 0.62 \\
\hline Newport & 3 & 0 & 0 & 3 & 0.46 \\
\hline typhi & 2 & 4 & 21 & 27 & 4.16 \\
\hline paratyphi $(A+B+C)$ & $1(B)+1(C)$ & $1(A)+17(B)+5(C)$ & $1(A)+2(B)$ & 28 & 4.31 \\
\hline Other five NTS serovars & 3 & 7 & 2 & 12 & 1.85 \\
\hline Total & 53 & 204 & 392 & 649 & 100 \\
\hline
\end{tabular}

Abbreviation: NTS, non-typhoid Salmonella.

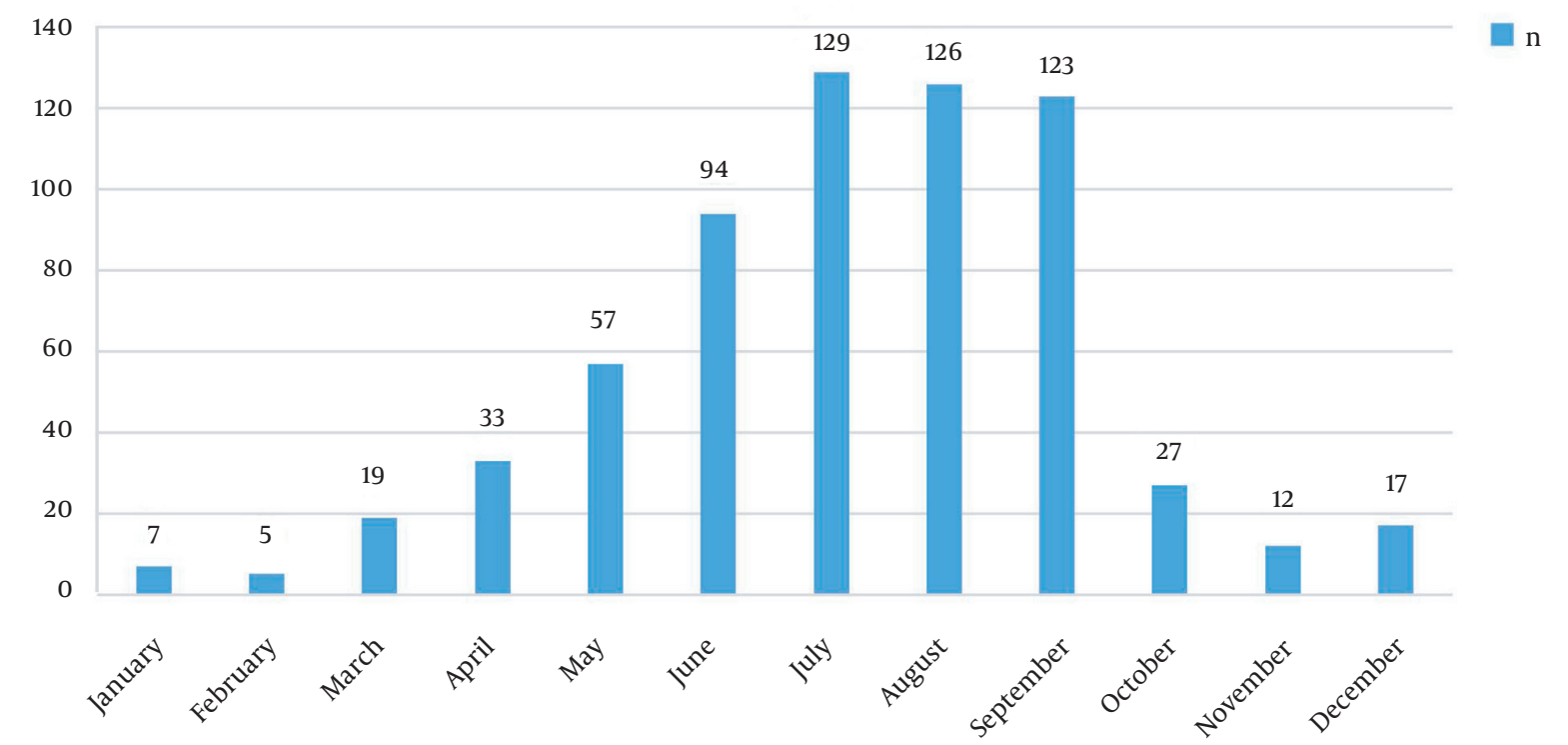

Figure 1. Monthly distribution of 649 Salmonella isolates in children with diarrhea in Chengdu, China, within 2018 - 2020

\subsection{Antimicrobial Susceptibility}

The isolated 649 enteric Salmonella strains had good susceptibility to cefotaxime and ciprofloxacin $(87.67 \%$ and $79.20 \%$, respectively), poor susceptibility to sulfamethoxazole (53.93\%), and almost no susceptibility to ampicillin. In addition, the drug resistance rate reached $92.91 \%$.

\section{Discussion}

In China, with the economic development and continuous improvement of sanitary conditions, the overall incidence of typhoid and paratyphoid fever has decreased; nonetheless, NTS is still on the rise, and the incidence of Salmonella in preschool children, especially children under 5 years of age, is very high (15). Salmonella typhimurium 
Table 2. Distribution of 649 Salmonella Isolates in Children with Diarrhea Regarding Gender in Chengdu, China, Within $2018-2020\left(\chi^{2}=4.106 ; \mathrm{P}=0.043\right)$

\begin{tabular}{lccc}
\hline Gender & $\begin{array}{c}\text { Number of } \\
\text { Specimens }\end{array}$ & $\begin{array}{c}\text { Number of } \\
\text { Salmonella }\end{array}$ & Prevalence, \% \\
\hline Male & 3767 & 343 & 9.11 \\
Female & 2889 & 306 & 10.59 \\
Total & 6656 & 649 & 9.75 \\
\hline
\end{tabular}

\begin{tabular}{lccc}
\hline $\begin{array}{l}\text { Table 3. Distribution of 649 Salmonella Isolates in Children with Diarrhea Regarding } \\
\text { Age in Chengdu, China, Within 2018-2020 }\left(\chi^{2}=392.96 ; \mathrm{P}<0.01\right)\end{array}$ \\
\hline Age Group & $\begin{array}{c}\text { Number of } \\
\text { Specimens }\end{array}$ & $\begin{array}{c}\text { Number of } \\
\text { Salmonella }\end{array}$ & Prevalence, \% \\
\hline 0-6 months & 3047 & 68 & 2.23 \\
\hline 7-12 months & 855 & 154 & 18.01 \\
\hline 1-3 years & 2068 & 351 & 16.97 \\
\hline 4-6 years & 359 & 50 & 13.93 \\
\hline 7-13 years & 327 & 26 & 7.95 \\
\hline Total & 6656 & 649 & 9.75 \\
\hline
\end{tabular}

as the main strain affected weak children under 2 years of age (15).

In this study, the positive rate of Salmonella in children with diarrhea under 3 years of age in Chengdu was $88.29 \%$, which is consistent with the results of previous studies, and $85.9 \%$ in Beijing (16). In children, owing to host factors, such as immature intestinal mucosal immune barrier, low gastric acid, weak autoantibodies, and absence of microfold (M) cells $(17,18)$, Salmonella invades intestinal epithelium, leading to intraepithelial proliferation and microcolony formation and reproduction, which are the main factors of Salmonella infection in children. Detection rates are the lowest under 6 months of age in children aged 1 day to 13 years with diarrhea, which is associated with the acquisition of maternal antibodies. Since children under 3 years of age are susceptible to Salmonella, this reminds us to protect this part of the population. In terms of gender, this study showed that there was no gender difference in Salmonella infection.

The distribution of $S$. enterica showed seasonality. Within January-June, with the continuous warming of the temperature, the infection of Salmonella in children increased gradually. In the hot summer and autumn (within June-September), Salmonella infection in children reached the highest level of $72.73 \%$ in the year, which is consistent with the results of previous studies (15). This finding is related to the subtropical monsoon humid climate in Chengdu. Salmonella-contaminated foods are more likely to spoil in warm wet summers and falls, increasing the risk of diarrhea in children. Accordingly, it is required to refrig- erate foods during the summer and fall to reduce the risk of Salmonella infection in children (19).

In this study, the resistance of Salmonella to Ampicillin was 92.91\%, which was much higher than the 33.3\% 64.8\% resistance of Salmonella CHINET (12) in China in 10 years. The other three antimicrobials were consistent with CHINET (12), showing high sensitivity to cefotaxime and ciprofloxacin and moderate sensitivity to sulfamethoxazole. Ampicillin and sulfamethoxazole are no longer suitable for clinical experience. Due to the high drug resistance of ampicillin and the high side effects and impacts of sulfamethoxazole and ciprofloxacin on children's health, Women's and Children's Central Hospital chose cefotaxime as the only treatment for Salmonella infection from the four antibiotics instead of these three drugs.

Due to time-consuming stool culture, children with serious infection symptoms will empirically choose cefotaxime, and the plan will be adjusted after the results of drug sensitivity. If cefotaxime is resistant, meropenem is the last option for children who have failed to respond to previous drugs and have developed intracranial infections. Antibiotic resistance in this study reflected the local bacterial drug resistance ecology, which is related to the fact that fungicides can act on multiple sites of microorganisms and cause bacterial drug resistance through nonspecific means (10). Rational drug use should be very urgent.

In this study, NTS in children was on the rise. The most important pathogenic factor of Salmonella infectious diarrhea in children in Chengdu was S. typhimurium rather than S. enteritis, which is consistent with CHINET (12), but inconsistent with the first-ranking of S. enteritis and the second-ranking of S. typhimurium in the world (11). The difference in serotype distribution might be related to the ecological environment and preventive measures in different countries and regions. Within 2018 - 2020, both 2019 and 2020 saw significant increases in the number of tests, compared to the previous year, particularly S. typhimurium. The possible reason is that the coronavirus disease 2019 (COVID-19) outbreak occurred for the first time in 2019, and individuals might pay more attention to the prevention and control of COVID-19 while ignoring food safety precautions. Due to this situation, it is equally important to strengthen children's dietary management.

For NTS, individuals and animals are its most extensive hosts. Humans acquire NTS infection through direct or indirect contact with animals or consumption of animal food. The NTS serotypes from humans might be related to endemic zoonotic serotypes (11). Studies have shown that four serotypes, including S. enteritidis, S. typhimurium, S. derby, and S. indiana, are frequently transmitted between humans and animals by infected animals and humans or 


\begin{tabular}{lcccc}
\hline Table 4. Susceptibility of 649 Salmonella enterica Isolates to Antimicrobial Drug & & & \\
\hline Antibiotics & Number & Susceptible & Intermediate & Resistant \\
\hline Ampicillin & 649 & $37(5.70)$ & $9(1.39)$ & $603(92.91)$ \\
Sulfamethoxazole & 649 & $350(53.93)$ & $59(9.09)$ & $240(37.00)$ \\
Ciprofloxacin & 649 & $514(79.20)$ & $64(9.86)$ & $71(10.94)$ \\
Cefotaxime & 649 & $569(87.67)$ & $25(3.85)$ & $55(8.47)$ \\
\hline
\end{tabular}

${ }^{\mathrm{a}}$ Values are expressed as No. (\%).

by contaminated animal foods (20). This is why it is very difficult to eradicate NTS in both developing and developed countries (2). According to a national sentinel survey (13), S. typhi and paratyphoid are generally at a low level in China; however, there are occasional outbreaks. In this study, the detection rates of S. typhi and paratyphi were lower than the national levels $(3.00 \%)$ of typhoid fever (13); nevertheless, the elimination of typhoid fever will take a long time.

Since humans are the only terminal host of S. typhi and paratyphi, the infection of typhoid to humans can be reduced by diagnosing and treating chronic typhoid and using vaccines to reduce the susceptibility of typhoid to the host (21). In developed countries, these preventive measures have largely eliminated enteric fever, which occurs mostly only in tourists returning from endemic areas (2224). However, in Asia and Africa, enteric fever is very prevalent due to economic impacts. paratyphoid $A$ is more severe in Asia, especially in China (25). Therefore, this is a serious problem.

China is a developing country in Asia. In the face of the increasing infection rate of NTS in children and no elimination of enteric fever, some measures need to be taken to control Salmonella infection in children. First, every Salmonella patient should be treated in the hospital until the gut is free of Salmonella. Drinking water resources are well protected to prevent sewers from mixing with drinking water. Moreover, the government should invest more in basic health facilities, and typhoid vaccination is advocated for children under 3 years of age and others.

Vaccines should focus on children under 5 years of age and be balanced against typhoid and paratyphoid; otherwise, the protection of typhoid vaccine against enteric fever might be reduced (21). At present, there are two kinds of licensed typhoid vaccines in the world. Due to economic reasons but considering the protective effect of typhoid vaccine on children, the China Government has designated typhoid vaccine as a class II vaccine and recommended that eligible children be vaccinated against typhoid. It is believed that Salmonella infection in children can be controlled through a combination of positive factors.

In this study, there were certain limitations regarding the methodology. On the one hand, due to the limitation of bacterial growth, it takes a long time for bacterial typing and drug sensitivity, which seriously affects the targeted clinical treatment of this disease. On the other hand, Salmonella with the same serotype might have different molecular types, which cannot be traced the source. At present, the bacteriophage typing technology is relatively mature, which can distinguish more than 300 Salmonella types with simple operation, high sensitivity, and short time. Moreover, it can trace the source of Salmonella infection outbreaks. It is suggested that time is needed to verify the feasibility of bacteriophage typing of Salmonella from scientific research to a clinical laboratory.

\subsection{Conclusions}

Salmonella typhimurium was the main Salmonella infection in children with diarrhea in Chengdu. The NTS and $S$. typhi infection are on the rise, and S. paratyphoid is unstable, presenting a phenomenon of transition and replacement. Salmonella infection was mainly in children under 3 years of age, with the highest infection rate within June to September. Salmonella had good sensitivity to cefotaxime and ciprofloxacin and a high drug resistance rate to ampicillin. It is necessary to strengthen rational drug use and Salmonella prevention measures.

\section{Acknowledgments}

First of all, the authors would like to express their gratitude to the Director of Gastroenterology Department of Chengdu, Women's and Children's Central Hospital, and her team for their efforts to carry out this study. Simultaneously, the authors would like to express their gratitude to the Leadership of the Laboratory, colleagues in the Microbiology Laboratory, and the examiner for the statistical data of this study.

\section{Footnotes}

Authors' Contribution: Jun-Ying Zhang carried out the design of the subject, prepared the statistics of the data, 
and drafted the manuscript. Wen-Juan Wang participated in the discussion. Xiao-Li Xie participated in the guidance of the project. Pei-Pei Song was involved in serotyping. Li-Hong Shang was involved in the clinical diagnosis and treatment of children with diarrhea. Li-Jing Xiong participated in the revision of the paper. Lin Deng participated in preparing the data statistics.

Conflict of Interests: The authors declare that they have no conflict of interest.

Ethical Approval: This study was conducted in accordance with the Declaration of Helsinki and approved by the Medical Ethics Committee of Chengdu Women's and Children's Central Hospital ([2013], Medical Ethics Committee, CWCCH). The study was retrospective, and the patients' names were intentionally removed; therefore, patients' informed consent was not required.

Funding/Support: This study was supported by the National Science and Technology Major Project during the 13th Five-Year Plan Period (code: 2017ZX10103010-006).

\section{References}

1. Ly KT, Casanova JE. Mechanisms of Salmonella entry into host cells. Cell Microbiol. 2007;9(9):2103-11. doi: 10.1111/j.1462-5822.2007.00992.x. [PubMed: 17593246].

2. Majowicz SE, Musto J, Scallan E, Angulo FJ, Kirk M, O'Brien SJ, et al. The global burden of nontyphoidal Salmonella gastroenteritis. Clin Infect Dis. 2010;50(6):882-9. doi: 10.1086/650733. [PubMed: 20158401].

3. Mogasale V, Maskery B, Ochiai RL, Lee JS, Mogasale VV, Ramani E, et al. Burden of typhoid fever in low-income and middle-income countries: a systematic, literature-based update with risk-factor adjustment. Lancet Glob Health. 2014;2(10):e570-80. doi: 10.1016/S2214109X(14)70301-8. [PubMed: 25304633].

4. Buckle GC, Walker CL, Black RE. Typhoid fever and paratyphoid fever: Systematic review to estimate global morbidity and mortality for 2010. J Glob Health. 2012;2(1):10401. doi: 10.7189/jogh.02.010401. [PubMed: 23198130]. [PubMed Central: PMC3484760].

5. Mogasale V, Mogasale VV, Ramani E, Lee JS, Park JY, Lee KS, et al. Revisiting typhoid fever surveillance in low and middle income countries: lessons from systematic literature review of population-based longitudinal studies. BMC Infect Dis. 2016;16:35. doi: 10.1186/s12879-016-13513. [PubMed: 26822522]. [PubMed Central: PMC4731936].

6. Antillon M, Warren JL, Crawford FW, Weinberger DM, Kurum E, Pak $\mathrm{GD}$, et al. The burden of typhoid fever in low- and middle-income countries: A meta-regression approach. PLoS Negl Trop Dis. 2017;11(2). e0005376. doi: 10.1371/journal.pntd.0005376. [PubMed: 28241011]. [PubMed Central: PMC5344533].

7. Als D, Radhakrishnan A, Arora P, Gaffey MF, Campisi S, Velummailum R, et al. Global Global trends in typhoidal salmonellosis: A systematic review. Am J Trop Med Hyg. 2018;99(3_Suppl):10-9. doi: 10.4269/ajtmh.18-0034. [PubMed: 30047364]. [PubMed Central: PMC6128363].

8. Wen SC, Best E, Nourse C. Non-typhoidal Salmonella infections in children: Review of literature and recommendations for management. J Paediatr Child Health. 2017;53(10):936-41. doi: 10.1111/jpc.13585. [PubMed: 28556448].

9. Uche IV, MacLennan CA, Saul A. A systematic review of the incidence, risk factors and case fatality rates of invasive nontyphoidal
Salmonella (iNTS) disease in Africa (1966 to 2014). PLoS Negl Trop Dis. 2017;11(1). e0005118. doi: 10.1371/journal.pntd.0005118. [PubMed: 28056035]. [PubMed Central: PMC5215826].

10. Goudarzi M, Navidinia M. Overview perspective of bacterial strategies of resistance to biocides and antibiotics. Arch Clin InfectDis. 2019;14(2). e6574. doi: 10.5812/archcid.65744.

11. Herikstad H, Motarjemi Y, Tauxe RV. Salmonella surveillance: a global survey of public health serotyping. Epidemiol Infect. 2002;129(1):1-8. doi: 10.1017/s0950268802006842. [PubMed: 12211575]. [PubMed Central: PMC2869853].

12. Xu YM, Du Y, Shan B, Wang CQ, Xue JC, Zhang H, et al. Resistance profile ofSalmonella isolates in hospital across China: results from CHINET Antimicrobial Resistance Surveillance Program, 20052014. Chin J Infect Chemother. 2016;16(3):294-301. doi: 10.16718/j.10097708.2016.03.009.

13. Cao Y, Han YY, Liu FF, Liao QH, Li J, Diao BW, et al. [Epidemiological characteristics and molecular typing of typhoid and paratyphoid in China, 2009-2013]. Chin J Epidemiol. 2018;39(3):337-41. Chinese. doi: 10.3760/cma.j.issn.0254-6450.2018.03.017. [PubMed: 29609250].

14. Shang H, Wang YS, Shen ZY. National Clinical Laboratory Procedures. 4 th ed. Beijing: People's health press; 2014. p. 560-752.

15. Zhu FT, Hu YM, Jiang ZF. Practical Pediatrics. 7th ed. Beijing: People's health press; 2005. p. 894-7.

16. Qu M, Huang Y, Zhang X, Lv B, Jia L, Tian W, et al. Characteristics of salmonella typhi and salmonella paratyphi strains in Beijing from 2008 to 2018. Capit J Public Heal. 2019;13(2):57-61. doi: 10.16760/j.cnki.sdggws.2019.02.015.

17. Shimoni Z, Pitlik S, Leibovici L, Samra Z, Konigsberger H, Drucker M, et al. Nontyphoid Salmonella bacteremia: age-related differences in clinical presentation, bacteriology, and outcome. Clin Infect Dis. 1999;28(4):822-7. doi: 10.1086/515186. [PubMed:10825045].

18. Zhang K, Dupont A, Torow N, Gohde F, Leschner S, Lienenklaus $S$, et al. Age-dependent enterocyte invasion and microcolony formation by Salmonella. PLoS Pathog. 2014;10(9). e1004385. doi: 10.1371/journal.ppat.1004385. [PubMed: 25210785]. [PubMed Central: PMC4161480].

19. Lake IR, Gillespie IA, Bentham G, Nichols GL, Lane C, Adak GK, et al. A re-evaluation of the impact of temperature and climate change on foodborne illness. Epidemiol Infect. 2009;137(11):1538-47. doi: 10.1017/S0950268809002477. [PubMed: 19371450].

20. Wu CM, Yan MY, Liu LZ, Lai J, Chan EWC, Chen S. Comparative characterization of nontyphoidal Salmonella isolated from humans and food animals in China, 2003-2011. Heliyon. 2018;4(4). e00613. doi: 10.1016/j.heliyon.2018.e00613. [PubMed: 29736431]. [PubMed Central: PMC5934692].

21. Gayet R, Bioley G, Rochereau N, Paul S, Corthesy B. Vaccination against Salmonella infection: the mucosal way. Microbiol Mol Biol Rev. 2017;81(3). doi: 10.1128/MMBR.00007-17. [PubMed: 28615285]. [PubMed Central: PMC5584317].

22. Gibani MM, Britto C, Pollard AJ. Typhoid and paratyphoid fever: a call to action. Curr Opin Infect Dis. 2018;31(5):440-8. doi: 10.1097/QCO.0000000000000479. [PubMed: 30138141]. [PubMed Central: PMC6319573].

23. Smith SI, Seriki A, Ajayi A. Typhoidal and non-typhoidal Salmonella infections in Africa. Eur J Clin Microbiol Infect Dis. 2016;35(12):1913-22. doi: 10.1007/s10096-016-2760-3. [PubMed: 27562406].

24. Connor BA, Schwartz E. Typhoid and paratyphoid fever in travellers. Lancet Infect Dis. 2005;5(10):623-8. doi: 10.1016/S1473-3099(05)70239-5. [PubMed: 16183516].

25. Ochiai RL, Acosta CJ, Danovaro-Holliday MC, Baiqing D, Bhattacharya SK, Agtini MD, et al. A study of typhoid fever in five Asian countries: disease burden and implications for controls. Bull World Health Organ. 2008;86(4):260-8. doi: 10.2471/blt.06.039818. [PubMed: 18438514]. [PubMed Central: PMC2647431] 